\title{
A new innate sensor for an ancient molecular pattern
}

\author{
LI LiWu \\ Department of Biological Sciences, Biomedical Engineering, and Medicine, Virginia Tech, Blacksburg, VA 24061-0910, USA
}

Received September 17, 2014; accepted September 22, 2014; published online October 10, 2014

Citation: Li LW. A new innate sensor for an ancient molecular pattern. Sci China Life Sci, 2014, 57: 1236-1237, doi: 10.1007/s11427-014-4761-8

Humans form a close co-habitat with Gram-negative bacteria since the beginning of ages. Perhaps one of the keys for human survival during this co-existence is the proper sensing and recognition of Gram-negative bacteria surrounding and within human tissues and cells. Lipopolysaccharide (LPS), also known as endotoxin, is a major component of the Gram-negative bacteria cell wall. Depending on the cell types and contexts, LPS can trigger a plethora of host responses that include the expression of pro- and anti-inflammatory cytokines, chemotaxis, phagocytosis, cell proliferation, necrosis and apoptosis, as well as maturation and differentiation of innate leukocytes such as macrophages, dendritic cells, and neutrophils. Due to its diverse and potent effects on host immunity, endotoxin recognition serves as a linchpin that underlies the proper immune defense against bacteria spread beyond mucosal barriers, chronic infection and inflammation, as well as acute cytokine storms and septic death.

Given its significance, decades of studies have focused on how host cells recognize bacteria LPS, and eventually led to the identification of an essential host cell membrane receptor, Toll-like-receptor 4 (TLR4). Not surprisingly, the discovery of TLR4 receptor was recognized by a recent Nobel prize [1]. A series of studies about TLR4 and its downstream signaling processes uncovered rich mechanisms responsible for the diverse cellular responses. However, intracellular bacteria must also be properly recognized and processed. Indeed, recent studies suggest that intra-cellular LPS may signal independently of TLR4, and induce the activation of a unique caspase, caspase $11[2,3]$.

email:1wli@vt.edu
Caspase 11 activation may enhance the host's ability to better process the inflammatory cytokines such as IL-1 $\beta$ and IL-18. In addition, this process may also lead to a distinct form of cell death termed pyroptosis. Despite these advances, the specific intracellular receptor for LPS has remained elusive until a recent study by Shi et al. [4]. To fill this critical void, this study from Dr. Shao Feng's group convincingly demonstrated that human caspase 4, a functional homologue of murine caspase 11, can directly interact with intracellular LPS in human cells, and responsible for the intracellular sensing of LPS.

By combined biochemical approaches that include the traditional pool-down assays and surface plasma resonance (SPR) measurements, the authors showed that both human caspase 4 and mice caspase 11 can form a high affinity complex with LPS or the lipid A moiety of LPS, but not MDP or Pam3CSK4 - other molecular patterns mimicking Gram-negative bacteria products. Furthermore, LPS binding induces oligomerization and activation of caspase 4/11. The penta-acylated LPS-RS, a functional LPS antagonist, can bind with caspase 11 with similar affinity, yet fails to induce caspase 4/11 oligomerization and activation. The authors also identified key lysine residues within caspase 11 necessary for the interaction with LPS. Another interesting finding is that caspase 5 can also interact with LPS, and offers a complex scenario of pyroptosis regulation by LPS.

At the functional level, Shi et al. [4] reported that LPS may activate caspase 4 in not only human macrophages, but also other non-immune cells such as epithelial cells and keratinocytes. As suggested by the authors in the report, this finding may open up novel functional relevance of LPS sensing by non-myeloid cells in the contexts of both acute 
and chronic infections.

This study by Shi et al. [4] not only elegantly solved the recognition issue of intracellular LPS, but also has far reaching clinical implications, since LPS is related to the pathogenesis of both acute and chronic inflammatory diseases. However, before we can fully harness the benefit of this finding, additional pressing questions must be addressed. First, it is not clear how cells properly coordinate the sensing of extra-cellular and intra-cellular LPS. Innate leukocytes are known to adopt dynamic responses to LPS, depending on LPS concentration and the prior cellular conditions, as reflected in the classical example of LPS priming and tolerance [5]. Future functional studies are warranted to clarify the systems dynamics of these molecular events regarding LPS recognition. Second, the regulations and roles of caspase 4/5/11 in different cell types are likely distinct. Detailed analyses are warranted to define the expression levels, downstream effector functions of caspase 4/5/11 in naïve and differentiated monocytes, macrophages, dendritic cells, neutrophils and B cells. The context-dependent function of LPS sensing by inter-cellular and extra-cellular sensor will bear critical relevance to the disease pathogenesis and homeostasis of tissue inflammation. Third, it remains to be determined whether caspase 4/5/11 may serve as the sensor for additional molecular patterns beside LPS. The extra-cellular sensor of LPS, TLR4 is known to mediate the sensing of other molecules such as taxol and fatty acids. There is a possibility that caspase 4/5/11 may also sense other self-molecules within cytosol. Addressing these issues will have clinical impacts in not only infectious diseases, but also inflammatory and auto-immune syndromes. Exciting questions and opportunities still await us focusing on LPS, an ancient molecule that has been accompanying humans for eternity.

1 Poltorak A, He X, Smirnova I, Liu MY, Van Huffel C, Du X, Birdwell D, Alejos E, Silva M, Galanos C, Freudenberg M, Ricciardi-Castagnoli P, Layton B, Beutler B. Defective LPS signaling in $\mathrm{C} 3 \mathrm{H} / \mathrm{HeJ}$ and C57BL/10ScCr mice: mutations in Tlr4 gene. Science, 1998, 282: 2085-2088

2 Hagar JA, Powell DA, Aachoui Y, Ernst RK, Miao EA. Cytoplasmic LPS activates caspase-11: implications in TLR4-independent endotoxic shock. Science, 2013, 341: 1250-1253

3 Kayagaki N, Wong MT, Stowe IB, Ramani SR, Gonzalez LC, Akashi-Takamura S, Miyake K, Zhang J, Lee WP, Muszyński A, Forsberg LS, Carlson RW, Dixit VM. Noncanonical inflammasome activation by intracellular LPS independent of TLR4. Science, 2013, 341: 1246-1249

4 Shi J, Zhao Y, Wang Y, Gao W, Ding J, Li P, Hu L, Shao F. Inflammatory caspases are innate immune receptors for intracellular LPS. Nature, 2014, doi: 10.1038/nature13683

5 Morris M, Li L. Molecular mechanisms and pathological consequences of endotoxin tolerance and priming. Arch Immunol Ther Exp (Warsz), 2012, 60: 13-18

Open Access This article is distributed under the terms of the Creative Commons Attribution License which permits any use, distribution, and reproduction in any medium, provided the original author(s) and source are credited. 